International Journal of Biomedicine | June 2019 - Volume 9, Issue Suppl_1: Abstracts From the Second Russian International Conference "Cryo-electron microscopy 2019: achievements and prospects"

\title{
ORAL ABSTRACT PRESENTATIONS
}

SESSION TITLE: COMPLEX AND EMERGING TECHNIQUES IN STRUCTURAL BIOLOGY

DOI: 10.21103/IJBM.9.Suppl_1.OR20

\section{Abstract OR-20: Visualization of Exosomes Using Transmission Electron Microscopy and Immunogold Labelling}

\author{
Dmitry Bagrov, Anna Vnukova, Evgeniy Evtushenko, Svetlana Semina \\ Faculty of Biology, Lomonosov Moscow State University, \\ Moscow, Russian Federation
}

Background: Extracellular vesicles are small membrane particles which are produced by the cells and released into the surrounding space. They can transfer biomolecules into recipient cells. The typical vesicle size is 100-1000 nm, so transmission electron microscopy (TEM) is the most suitable tool for their visualization. This is especially true for the smallest vesicles - the exosomes with the size below $200 \mathrm{~nm}$.

Methods: We isolated exosomes produced by MCF-7 cancer cells and the two drug-resistant MCF7 sublines (Semina et.al., Molecules, 2018). The exosomes were isolated from the conditioned medium by ultracentrifugation, and their size was measured by nanotracking analysis (NTA) and TEM. Immunogold labelling was carried out on exosomes deposited onto carbon TEM grids. It involved the following principal steps: the vesicle deposition, sample treatment by the anti-CD9 antibodies, sample treatment by the $10 \mathrm{~nm}$ colloidal gold nanoparticles conjugated with protein A, staining with uranyl acetate. Washing and blocking steps were done between the principal ones. The samples were imaged using a JEM-1011 microscope at $80 \mathrm{kV}$.

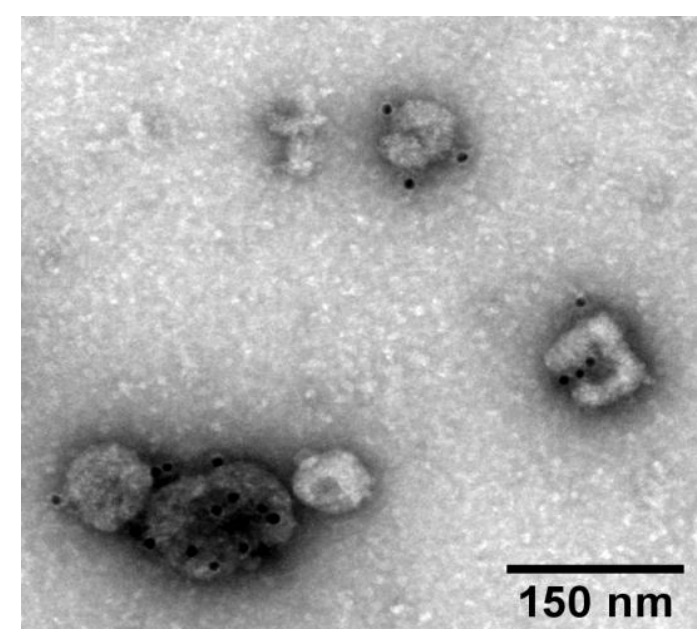

Fig. 1. Exosomes produced by the MCF-7 cells, labelled with colloidal gold. 
Results: NTA and TEM yielded similar results - the exosomes produced by the three studied cell lines had the almost equal size in the range from 50 to $200 \mathrm{~nm}$. When imaged by TEM, most exosomes demonstrated the typical cup-shaped morphology. We used immunogold labelling to verify that the exosomes carried the CD9 marker on them (Fig. 1). We achieved the labelling specificity in the range of $8-10$; this value was calculated as the ratio of the surface densities of the exosome-bound labels (colloidal gold nanoparticles) and the non-specific background ones.

Conclusion: We hope that the sample preparation and imaging procedures that we used could be useful for the investigation of other extracellular vesicles.

Key Words: exosomes $\cdot$ nanotracking analysis $\cdot$ transmission electron microscopy $\cdot$ immunogold labeling

Sources of Funding: The TEM measurements were performed at the User Facilities Center "Electron microscopy in life sciences" at Lomonosov Moscow State University. The work was supported by RFBR (Project 18-504-12045).

International Journal of Biomedicine. 2019;9 Suppl 1: S14. doi: 10.21103/IJBM.9.Suppl_1.OR20

C2019 International Medical Research and Development Corporation 